Orbit of $\Sigma 2$.

The observations of $\Sigma_{2}$ are neither numerous nor very consistent. For the period $1872-1884$ one is compelled to rely entirely upon the results obtained by $O$. Struve, but it has been necessary to increase the position angles in 1875 and 1876 by $180^{\circ}$. The near equality in magnitude of the stars justifies this step. Doberck's position angles for 1877-78 cannot be reconciled with the rest of the observations and have been disregarded. Measurements during the period I 894-1904, which would have helped very greatly to an accurate determination of the elements, unfortunately are wanting. According to the ephemeris the separation of the stars at the present time is about $O^{\prime \prime} I$, but in six years will have increased to o." 3 .

I find the following elements:

$$
\begin{array}{ll}
T=1902.60 & \lambda=34^{\circ} \mathrm{I} \\
P=128.55 & \gamma=77.4 \\
\mu=-2.8005 & \delta=166.6 \\
\iota=0.392 & a=0.612
\end{array}
$$

\begin{tabular}{|c|c|c|c|c|c|}
\hline Observer & Date & $\theta_{c}$ & $\varrho_{c}$ & $\theta_{o}-\theta_{c}$ & $\varrho_{o}-\varrho_{c}$ \\
\hline $\mathbf{\Sigma}$ & I 828.25 & $341 \% 9$ & 0.79 & $+10 I$ & +0.01 \\
\hline$h$ & 183 & 34 I.2 & 0.7 & -1.5 & -0.08 \\
\hline$\Sigma$ & 1832.59 & 340.4 & 0.76 & +0.1 & +0.05 \\
\hline Dawes & I 83 & 33 & 0.7 & -1.5 & 0.00 \\
\hline$O \Sigma$ & 1840. & $33^{8.4}$ & 0.69 & +1.2 & +0.05 \\
\hline Mädler & 1841.57 & $33^{6.7}$ & 0.68 & -0.4 & -0.02 \\
\hline Mädler & 1842.63 & 3.36 .2 & 0.66 & $-\mathrm{r} .6$ & -0.02 \\
\hline Mädler & I 843.68 & 335.8 & 0.65 & +0.7 & -0.04 \\
\hline
\end{tabular}

$\lambda$ is measured from the node in the direction of motion.

Rectangular coordinates are given by

\begin{tabular}{|c|c|c|c|c|c|}
\hline Observer & Date & $\theta_{c}$ & $\varrho_{c}$ & $\theta_{o}-\theta_{c}$ & $\varrho_{o}-\varrho_{c}$ \\
\hline$O \Sigma$ & & $333^{\circ} \cdot 2$ & 57 & +1.6 & -0.05 \\
\hline Mädler & & & & $+8 . \mathrm{I}$ & -0.04 \\
\hline$O \Sigma$ & 6 & & & +3.9 & +0.02 \\
\hline $2 u n$ & & $325 \cdot 3$ & $0.4 \mathrm{I}$ & -0.4 & -0.03 \\
\hline & 9 & 322.2 & 0.36 & +4.6 & +0.08 \\
\hline Mädler & & $3 \times 6.9$ & $0.3 \mathrm{I}$ & +3.5 & -0.01 \\
\hline$D e$ & 186 & 309.1 & 0.26 & -13.6 & +o:1 2 \\
\hline Talma & I 86 & 308.8 & 0.26 & -13.2 & +0.04 \\
\hline Secchi & & 304.5 & 0.23 & +12.3 & +0.02 \\
\hline Duner & I 86 & 292 & 0.19 & +37.9 & -0.09 \\
\hline$O \Sigma$ & & 270 & 0.17 & - I $7:$ & - \\
\hline OY & & 242 & 0. & +1.8 & -- \\
\hline o. & I 87 & 229.5 & 0.16 & +5.8 & - \\
\hline$O \Sigma$ & I 87 & 2 I 0.4 & 0.18 & +5.6 & 一 \\
\hline$O \Sigma$ & & I 89.1 & 0.25 & $-3 \mathbf{I}:$ & - \\
\hline Tarra & I 88 & I 80.3 & 0.33 & +2.5 & -0.03 \\
\hline Hall & I 888 & I 78.7 & 0.35 & +4.9 & -0.02 \\
\hline$\beta$ & & 177.4 & 0.36 & -3.0 & -0.01 \\
\hline$H \Sigma$ & 1890.29 & I 76.7 & 0.36 & -.2 .3 & +0.03 \\
\hline$\beta$ & .82 & 175.8 & 0.37 & +1.3 & -0.08 \\
\hline$\beta$ & & 173.5 & 0.38 & -5.2 & -0.08 \\
\hline & & 172.6 & $0.3^{8}$ & -5.1 & +0.05 \\
\hline Comstock & I 893.94 & 171.5 & 0.39 & $-\quad 2.8$ & - O. I I \\
\hline Biesbroeck & 1904.10 & I 55.0 & 0.27 & -2.3 & +0.07 \\
\hline
\end{tabular}

$$
\begin{aligned}
\varrho \cos \theta=x & =[9.7630]^{\prime \prime} \cos \left(E+\mathrm{I} 45^{\circ} .20\right)+0.1 \mathrm{1} 865 \\
\varrho \sin \theta=y & =[9.2835]^{\prime \prime} \cos (E+7.72)-0.0746 \\
z & =\left[9.75^{21}\right]^{\prime \prime} \cos (E-\mathrm{I26.35})+0.1313 .
\end{aligned}
$$

London, Kings College, I 913 May 7 .
Ephemeris.

\begin{tabular}{rrc|rcc} 
Date & \multicolumn{1}{c|}{$\theta$} & $\rho$ & Date & $\theta$ & $\rho$ \\
1910 & 108.40 & \multicolumn{1}{c|}{0.10} & 1935 & $349^{\circ} .77$ & 0.68 \\
1915 & 21.08 & 0.17 & 1940 & 347.70 & 0.74 \\
1920 & 2.64 & 0.33 & 1945 & 345.99 & 0.79 \\
1925 & 356.07 & 0.48 & 1950 & 344.19 & 0.80 \\
1930 & $35^{2.37}$ & 0.59 & & &
\end{tabular}

F. B. Dale.

\title{
L'éclat de la comète 1907 IV (Daniel).
}

Pour définir l'éclat moyen de la comète I907 IV je me suis servi des observations de Holetschek du 17 juin jusqu'au 30 août publiées dans A. N. $175 \cdot 305$ et 177.99. Jusqu'à la mi-juillet la courbe de l'éclat de la comète monte rapidement, à partir de la mi-juillet la loi du changement de l'éclat varie brusquement et la courbe devient sensiblement déclive. Il est intéressant de noter ici que le 27 juin d'après Wolfer il y avait un minimum partiel des $\gg$ Relativzahlen« du soleil, exprimé par le nombre 8 , après lequel la courbe "Relativzahlen " monte brusquement et juste au milieu de juillet a le maximum partiel Io3. La courbe est restée approximativement à la hauteur de 60 , jusqu'à Ja fin du mois d'aout, avec des fluctuations presque insensibles en haut et en bas.

J'ai mis à part dans un groupe spécial les six premières observations de Holetschek, du I 7 juin jusqu'au 5 juillet inMoscou, Observatoire Archinoff, 19 3 Février. clusivement, car la loi du changement de l'éclat n'a pas été ici tout-à-fait la même. Nous obtenons par le procédé habituel $\left.^{1}\right)$ les équations qui réunit l'éclat moyen de la comète $\left(H_{0}\right)$ et l'exposant $n$ de la puissance du $r$. En résolvant ces six équations par le procédé de moindres carrés j'ai obtenu les normales suivantes.

$$
6 x+1.033 y=41.06 \quad 1.033 x+0.188 y=7.266
$$

où $x=H_{0}$ et $y=2.5 n$ d'où $H_{0}=3^{\mathrm{m}} \cdot 6 \pm 0^{\mathrm{m}} \cdot{ }^{\circ} 4 ; n=7.6$.

Le second groupe des observations à partir du 5 juillet jusqu'au 30 août donne les normales suivantes.

$24 x-2.485 y=72.59-2.485 x+0.526 y=-5.684$ d'où $\quad H_{0}=3^{\mathrm{m}} \cdot 7 \pm 0^{\mathrm{m}} \cdot \mathrm{o}_{2} \quad n=2.7$.

Les neuf observations de Holetschek sur l'éclat du noyau de la comète donnent les normales suivantes

$9 x+1.021 y=70.70 \quad$ 1.021 $x+0.162 y=8.199$

d'où $\quad h_{0}=7^{\mathrm{m}} \cdot 4 \pm 0^{\mathrm{m}} \cdot \mathrm{o}_{5} \quad n=$ 1.6.

S. Orlow. 